\title{
Total Knee Replacement: 12 Years Retrospective Review and Experience
}

\author{
Ahmad Hafiz Z, MS Orth, Masbah 0*, MS Orth, G Ruslan**, MS Orth \\ Department of Orthopaedics, Traumatology and Rehabilitation, \\ International Islamic University Malaysia, Kuantan, Malaysia \\ *Department of Orthopaedic and Traumatology, University Kebangsaan Malaysia Hospital, \\ Kuala Lumpur, Malaysia \\ **Tawakal Hospital, Kuala Lumpur, Malaysia
}

\begin{abstract}
This retrospective study was undertaken to evaluate the outcome of primary total knee arthroplasty (TKA) performed from January 1986 to December 1997 at this institution. Case review included Knee Society scores and functional knee score. The records of 94 patients (128 knees) were available for analysis with the sample comprised of 76 females (80.9\%) and 18 males (19.1\%) and a mean age was 61.4 years. TKA was performed for osteoarthritis (OA) in 96 knees (75\%) and rheumatoid arthritis (RA) in 32 knees $(25 \%)$. Cemented TKAs were performed in all patients. The mean knee score improved from 38.8 preoperatively to 90.9 postoperatively. The mean functional score improved from 19.1 preoperatively to 62.5 postoperatively. Both scores showed significant improvement when comparing preoperative to post operative results $(\mathrm{p}<0.005)$. Arthroplasty was designated failures if the prostheses used had been removed. Survivorship at 12 years was $89.5 \%$.
\end{abstract}

Key Words:

TKA, Osteoarthritis, Knee Score

\section{INTRODUCTION}

Total knee arthroplasty (TKA) represents a major advance in the treatment of degenerative joint disease, providing excellent restoration of joint function and pain relief. In Malaysia, TKA has likely been performed since the late 1970s. Dhillon et al reported on cases performed at University Hospital Kuala Lumpur since 1986, and has been undertaken with increasing frequency since that time ${ }^{1}$. The present study was conducted to retrospectively review retrospectively the results of all primary TKA performed at Hospital Kuala Lumpur over a period of 12 years from January 1986 to December 1997. The main aim of the study were analyse knee and functional score outcomes of patients following TKA. Knee scores and functional scores of the primary TKA were performed pre- and post-operatively.

\section{MATERIALS AND METHODS}

This retrospective study was undertaken to evaluate the outcome of TKA performed in Kuala Lumpur Hospital from January 1986 to December 1997. Inclusion criteria consisted of: surgery performed by the senior author, presence of a complete record and follow-up for at least 1 year. A total of 225 primary TKAs were performed and the records of 128 knees were available for analysis. This represents $56.9 \%$ of the primary TKAs that were performed during twelve years period. The low numbers of cases available was mostly due to the fact that several patients were referred from outlying hospitals and were followed up at their respective local hospital.

The Knee Society rating score system was used, and knee scores and functional scores were calculated separately. The knee score consist of pain, range of motion and stability domains whereas the functional score is used to assess the ability of the patient to walk, climb stairs and whether aids are required for walking. The total of 100 points was given to each score. Comparison between preoperative score and postoperative score obtained on the last follow-up was made. Knee score and functional score (2) of 85 to 100 points were designated as excellent results, 70 to 84 points a good result, 60 to 69 points as fair result and below 60 points as a poor result $^{2,3}$.

Statistical analysis of both knee scores and functional scores were compared using SPSS software, Version XXXX for Windows. Survivorship analysis was compared using the Kaplan-Meier method ${ }^{4}$. Arthroplasty was designated as a failure if prosthetic component were removed or added during the follow-up period. The remaining arthroplasties were regarded as successful (or non-failures) regardless of complications and clinical score ${ }^{5,6,7}$. 


\section{RESULTS}

The study sample consisted of 94 patients (128 Knees), 18 males (19.1\%) and 76 females (80.9\%). Osteoarthritis was diagnosed in 96 knees (75\%) and rheumatoid arthritis in 32 knees $(25 \%)$. Thirty-four patients underwent bilateral procedures and 60 a single sided procedure. Ethnic makeup was: 68 (53.1\%) Chinese, 28 (21.9\%) Indian, 22 (17.2\%) Malay, 9 (7.0\%) Sikh, and 1 Gurkha patient (0.8\%). Chinese females comprised the largest subgroup. (61 knees / 47.7\%) (Figure 1).

The average weight of study subjects was $63.7 \mathrm{~kg}$ (range, 40 to $93 \mathrm{~kg}$ ), $57.1 \mathrm{~kg}$ for rheumatoid patients (range, $40-75 \mathrm{~kg}$ ), $66.0 \mathrm{~kg}$ for osteoarthritis (range, $47-93 \mathrm{~kg}$ ). The follow-up period ranged from 3 to 12.8 years (mean, 64 months). The average age of the patients was 61.4 years (range, 37 to $80 \mathrm{y}$ ); $41.4 \%$ of patients were below 60 years old and $58.6 \%$ were $60 \mathrm{y}$ and above.

All patients were prescribed prophylactic antibiotics prior to surgery and continued post operatively ( 2 doses per day) for 3 days. Antibiotics used were Cefoperazone (51.1\% / 59 cases), Cefuroxime (45.3\% / 58 cases), Claforan (4.7\% / 6 cases) and Rocephine (3.9\% / 5 cases). Prophylaxis for thromboembolic prevention was only started in one patient who had a history of previous episode of deep venous thrombosis (DVT).

All surgeries were performed by an orthopaedic surgeon and assisted by postgraduate trainees or / and house officers. Tourniquets were applied in all cases and surgical approach was midline straight incision with medial parapatellar incision in all cases. Cemented TKA was performed in all patients. The Insall-Burstein (IB) system was the most commonly used $(41.4 \%$ or 53 knees) followed by press fit condylar (PFC, Sigma) (16.4\% or 21 knees), Whiteside Ortholoc ( $10.2 \%$ or 13 knees $),$ AGC $(6.3 \%$ or 8 knees $)$, AMK (5.5\% or 7 knees), Genesis ( $6.3 \%$ or 3 knees), Miller Galante, Osteonic and Rotaflex system $(0.8 \%$ or 1 patient $)$ respectively (Figure 2). A drain was inserted in all cases and was removed within 48 hours. Procedures were most commonly performed on the left knee (52.3\%) compared to right knee $(47.7 \%$ or 61 knees). Duration of the surgery ranged from 65 minutes to 360 minutes (Mean, $63.8 \pm$ 44.75).

Pain, a significant component of the knee score, improved significantly $(\mathrm{p}<0.005)$ after TKA. Preoperative pain was $9.92 \pm 5.81$ points while postoperative pain was rated at $46.91 \pm 3.08$ points. Stability improved significantly after TKA $(\mathrm{p}<0.005)$. Preoperative stability was $9.19 \pm 12.42$ points, and improved postoperatively to $24.32 \pm 3.04$ points. Range of motion was $97.58^{\circ} \pm 22.47$ (range $10^{\circ}$ to $135^{\circ}$ ) prior to surgery and postoperatively it increased to $99.77^{\circ} \pm 12.50$ (range, $30^{\circ}$ to $120^{\circ}$ ), not a statistically significant change $(\mathrm{p}>$ $0.05)$.
Preoperative functional knee scores were uniformly poor and improved postoperatively with excellent results in 3 knees $(2.4 \%)$, good in 62 knees $(48.4 \%)$, fair 25 knees (19.5\%), and poor in 38 knees $(29.7 \%)$. Thus, excellent and good results were achieved in $50.8 \%$ of the cases (65 of 128 knees). Knee scores and functional scores improved significantly improved on the last follow-up compared to preoperative assessments $(\mathrm{p}<0.005)$. The mean preoperative knee score was $38.77 \pm 15.68$ (range 0 to 65 points), while the mean post TKA knee score was $90.94 \pm 5.65$ (range, 56 to 99 points). The mean preoperative functional score was $19.10 \pm 13.89$ (range, 0 to 50 points), while mean post TKA functional scores were $62.54 \pm 16.68$ (range 5 to 90 points). Paired student $\mathrm{T}$ test analysis showed significant improvement between pre- and post-operative TKA results $(\mathrm{p}<0.005)$. For knee scores, 6 knees $(4.7 \%)$ were fair preoperatively and another 122 knees $(95.3 \%)$ were poor. Post TKA results were excellent in 120 knees (93.7\%), good in 6 knees $(4.7 \%)$, fair in 1 knee $(0.8 \%)$ and poor in 1 knee $(0.8 \%)$. Excellent and good results were achieved in $98.4 \%$ of cases.

Mean walking score preoperatively was $9.38 \pm 4.82$ points, and improved postoperatively to $31.02 \pm 10.75$ points $(p<0.005)$. The capability to climb stairs also improved significantly after TKA $(\mathrm{p}<0.005)$. Preoperatively the mean stair climbing scores were $11.21 \pm 10.75$ and improved postoperatively to $32.58 \pm 7.15$ points. The need to use a cane or walking stick sticks to assist walking also improved following surgery $(\mathrm{p}<0.05)$.

Osteoarthritis knee was noted in 87 knees in category A (a unilateral or successful bilateral knee replacement), 9 knees in category B (symptoms in the contralateral knee), but none from category $\mathrm{C}$ (associated medical conditions that limited function) $)^{2,8}$. There were 9 knees with rheumatoid arthritis, 9 in category A, 9 in category B and 14 in category C. (Table 1)

There were 96 TKA (75.0\%) in category A, 18 TKA (14.1\%) in category $\mathrm{B}$, and 14 TKA $(10.9 \%)$ in category $\mathrm{C}$. There was no significant difference in the duration of hospital stay between the two methods of physiotherapy (continuous passive motion or static quadriceps exercises) prescribed for study patients. The Chi-square test showed $\mathrm{p}=0.298$ $(\mathrm{p}>0.005)$.

\section{Complications}

Twelve knees had one or more complications. These included superficial wound infection (5knees, 3.9\%), deep infection (5knees, 3.9\%), knee instability ( 1 knee, $0.8 \%$ ), and polyethelene wear (thinning of the implant) (1 knee, $0.8 \%$ ).

One patient had deep infection 6 months after the procedure. Another 4 were diagnosed with deep infection approximately 2 years after undergoing TKA. All of these patients had osteoarthritis prior to surgery. Two had comorbid medical conditions, one with ischemic heart disease and another with 
Table I: Knee scores and functional scores preoperative and postoperative.

\begin{tabular}{|llllll|}
\hline Category & No. & Preop KS 1 & Postop KS 2 & Preop FS 1 & Postop FS 2 \\
\hline A & 96 & $39.75 \pm 15.56(0$ to 65$)$ & $91.10 \pm 5.57(56$ to 99$)$ & $21.41 \pm 13.29$ (0 to 50) & $63.96 \pm 14.67(25$ to 90$)$ \\
B & 18 & $37.56 \pm 19.08(0$ to 57$)$ & $91.56 \pm 6.48(69$ to 97$)$ & $15.00 \pm 12.00$ (0 to 35) & $59.17 \pm 20.95$ (5 to 80) \\
C & 14 & $33.64 \pm 11.31(19$ to 57$)$ & $89.67 \pm 5.11$ (77 to 97) & $8.57 \pm 14.99$ (0 to 50) & $57.14 \pm 22.59$ (20 to 90 \\
\hline
\end{tabular}

$\mathrm{KS}=$ Knee score, $\mathrm{FS}=$ Functional score

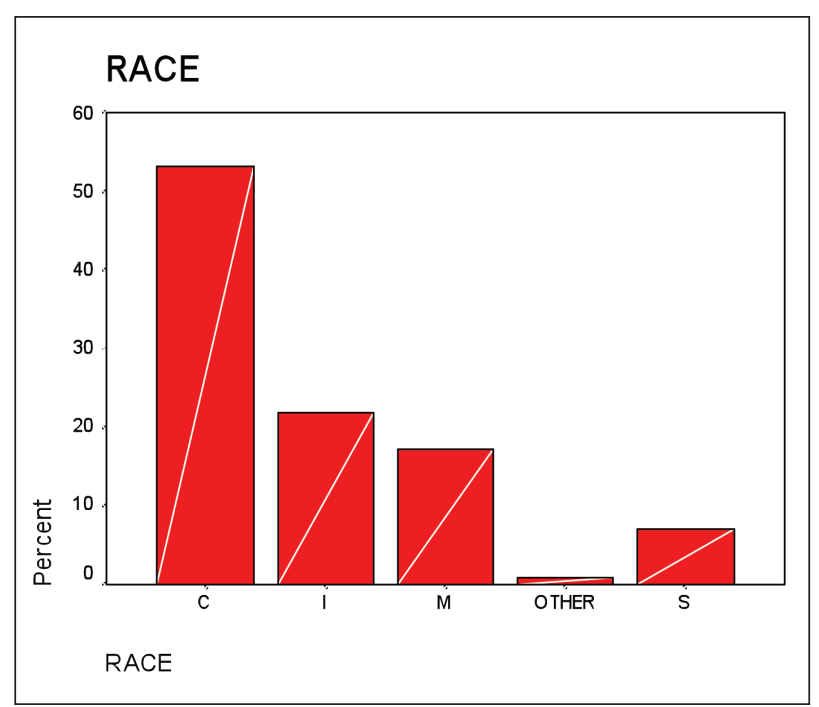

Fig. 1: Race distribution among the patients.

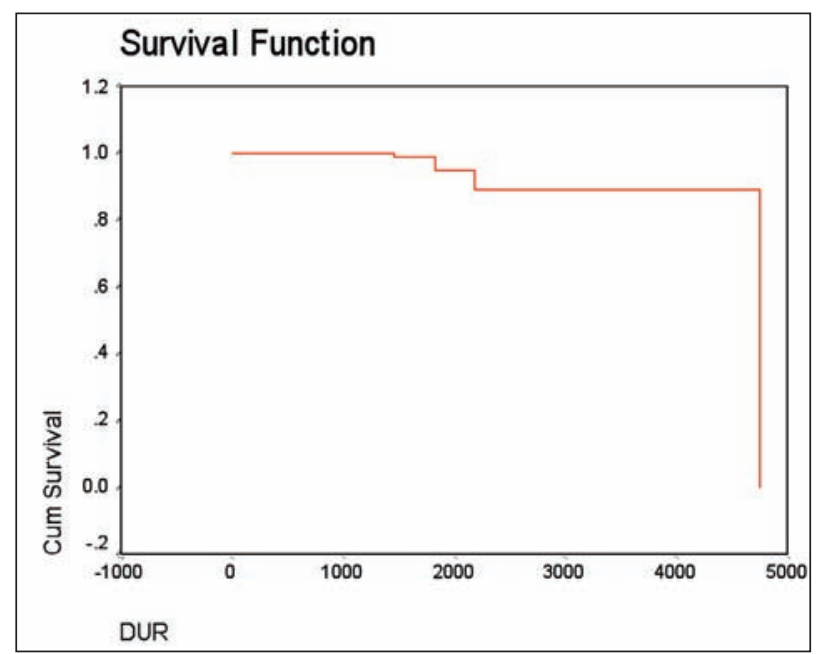

Fig. 3: ival analysis showed $89.2 \%$ survival at 12 years.

hypertension. The most common organism cultured was Methicillin resistance Staphylococcus aureus (MRSA), was found in $80 \%$ of cases (4 patients). The other patient had enterobacter and Staphylococcus aureus infection, which was sensitive to IV Cefoperazone. This patient developed a second infection one year later, in which Enterococci was isolated. Multiple debridements were performed and

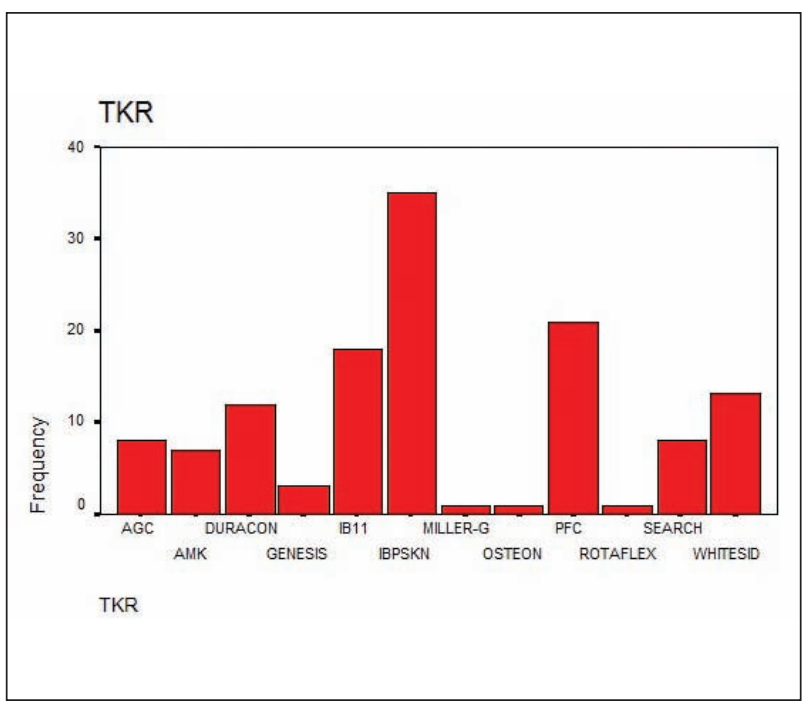

Fig. 2: Type of implant used.

removal of the implant was indicated due to persistent infection. Two patients had arthrodesis of the knee and three patients had a bone cement spacer inserted and were planned for revision surgery. Deep infection leads to poor postoperative results.

Superficial wound infection was noted in 5 TKAs (3.9\%), and all of these patients responded to intravenous antibiotics. Amongst these five patients, three s had osteoarthritis and another two, rheumatoid arthritis. Organism isolated were: Methicillin-resistance Staphylococcus aureus (MRSA) in $60 \%$ of the cases (3 patients); Streptococcus group D in $20 \%$ of the cases (1 patient); and Staphylococcus aureus in another $20 \%$ of cases (1 patient). Patients with MRSA infection were treated with parenteral antibiotics consisting of Fucidic acid, Vancomycin and Ciprofloxacin. Elase and Oxoferin dressing were also used. The Streptococcus group $D$ (one patient) was sensitive to IV Cloxacillin and responded well to parenteral antibiotic therapy. Staphylococcus aureus which was isolated in another patient also was sensitive to IV Cloxacillin. This patient responded well after debridement, parenteral antibiotic therapy and secondary suturing. In cases of superficial infections, the TKA survived with aggressive debridement and antibiotics. 
One patient $(0.8 \%)$ developed subluxation of the TKA due to instability. A $16 \mathrm{~mm}$ tibial insert was used in the first surgery. Subluxation was noted after day 3 postoperatively and the patient also developed superficial wound infection postoperatively. Closed manual reduction was attempted under guidance of Image Intensifier but with no success. The patient then underwent a second operation after the superficial wound infection (Staphylococcus aureus was isolated and sensitive to IV Cloxacillin) was treated and healed. The tibial insert size was changed to $20 \mathrm{~mm}$.

One patient was noted to have polyethylene thinning at six years postoperatively. This patient had osteoarthritis secondary to trauma prior to the operation. Revision surgery was performed 6 years after the primary TKA. This was the only one revision $(0.8 \%)$ done during the period of this study. Revision was performed as there is no similar system available to replace the thinning polyethylene. This patient underwent TKA in 1993 using the Whiteside Ortholoc System; Insall Burstein II System was used for the revision surgery and a $12 \mathrm{~mm}$ tibial insert was used.

Survivorship analysis is the preferred method for comparing different series using the same prostheses or series of different prostheses $6,7,9,10$. Wstimated survivorship for this study at 12 years is $89.5 \%$ (Confidence Interval, 95\%). Survivorship graphs were constructed using 1000 days intervals. Survivorship at 5 years is $95 \%$. (Figure 3)

\section{DISCUSSION}

This study was undertaken to review initial TKA experience at a single hospital. The majority of patients undergoing TKA in the present study were females $(80.9 \%)$ and the preoperative diagnosis was osteoarthritis in 96 knees $(75 \%$ of the knees) and rheumatoid arthritis in 32 knees (25\%). This is similar to reported results by Dhillon et al where the majority of their patients were females (76\%) and preoperative diagnosis was osteoarthritis in $81 \%$ of the cases $^{1}$. Schurman et al reported $69.1 \%$ cases were females and osteoarthritis was noted in $45.1 \%$ of the cases and rheumatoid arthritis in $46.2 \%$ of cases ${ }^{1}$. Kuntson et al reportedosteoarthritisin $56.3 \%$ of cases, a majority of participants were women ${ }^{3}$. Schai et al reported $62 \%$ of their patient had knee osteoarthritix and 33\% with rheumatoid arthritis (RA) ${ }^{10}$. Ranawat et al reported that most of their patient were female $(80 \%, 68$ of 85 patients $)$ and osteoarthritis was present in $44.6 \%$ of their sample (50 of 112 knees) while rheumatoid arthritis was noted in 53.4\% (62 of 112 knees) of cases ${ }^{12}$.

The mean age in this study was 61.4 years (range, 37 to $80 y$ ). Most other studies reported the mean age of patient in their studies in the sixth decade of life: (Stern and Insall, an average age was 63 years (range, 17 to $87 \mathrm{y}$ ); Scott et al, an average age of 66.9 years (range 22 to $85 \mathrm{y}$ ); and Schurman et $a l$, and average age of 63.5 years ${ }^{7,8,11}$. On the other hand, younger average ages were reported by: Duffy et al, age was 43 years (range $10-17$ years); Diduch et al, average age of 51 years (range 22 to 55 years); and, Ewald et al, average age of 76 years (range 25 to 86 years) ${ }^{13,14,15}$. Locally, Dhillon et al reported an average age of 66.5 years (range 21 to 85 years) ${ }^{1}$.

The ranges of motion (ROMs) reported includes Ranawat et al,mean postoperative $\mathrm{ROM}$, was $99^{\circ}$ (range, $65^{\circ}-120^{\circ}$ ). ) as well as Duffy et al, mean preoperative ROM, $110^{\circ}$ (range, $0 \mathrm{o}$ to $135 \mathrm{o}$ ) and postoperative mean ROM, 100o (range 35o $\left.-135^{\circ}\right)^{13,16}$. In the present study, the preoperative mean ROM was $97.580 \pm 22.47$ (range, 10 to $135^{\circ}$ ) and postoperative $\mathrm{ROM}$ mean was $99.77^{\circ} \pm 12.50$ (range 30 to $120^{\circ}$ ). We note that most of patient had reduced range of motion pre-operatively, and some continued to have less than optimal ROM postoperatively. However, ROM improved in most patients with good range of motion prior to surgery. Although soft tissue analysis is another area involved in overall ROM outcome, this is not covered in the scoring system used here and in other reports

Overall, he knee scores and functional scores significantly improved after TKA $(\mathrm{p}<0.005)$. In this study, $98.4 \%$ of knee scores were either excellent or good, an outcome that is similar to other studies. For instance, the following reported good or excellent results: Ewald et al, 90\% (111 knees); Diduch et al, 100\% (103 knees); Ranawat et al, 92\% (103 knees); Scott et al reported 98\% (116 knees) were good $(15 \%)$ or excellent $(83 \%)$ with a posterior substituting prosthesis ${ }^{7,12} 14,116,7$. Duffy et al) reported significant improvement of knee score postoperatively $(\mathrm{p}<0.001)$ (preoperative knee score of 36 points (range $10-80$ points) compared to 84 points (range $37-100$ points) postoperatively ${ }^{13}$. Dhillon et al reported 78\% (39 of 50 knees) achieved good and excellent result ${ }^{1}$. Brander et al reported outcome of hip and knee arthroplasty in persons aged 80 years and older ${ }^{18}$. For instance, they noted that the mean knee score preoperatively was 58 and improved postoperatively to a mean score of 77 . Pain improved in $98 \%$ (total patients of 51 with 56 TKAs) of TKAs. Callahan et al noted good and excellent outcome varied from $52 \%$ to $100 \%$ amounts in the meta-analysis of 57 studies ${ }^{19}$. In 26 studies, $75 \%$ of patients reported no pain postoperatively, $20 \%$ reported mild pain, $3.7 \%$ moderate pain and $1.3 \%$ reported severe pain. Thus, overall, our knee score results were similar when compared to reported studies by other authors.

Functional scores also showed significant improvement after TKA $(\mathrm{p}<0.005)$. The mean preoperative was $19.10 \pm 13.89$ (range 0-50 points) while the post TKA functional score mean was $62.54 \pm 16.68$ (range, 5- 90 points). Similarly, Duffy reported similar significant improvement from preoperative functional mean score of 45 points (range, 0 - 
100 points) to a postoperative mean of 60 points (range, 0 100 points $)(p<0.001)^{13}$. The functional score was excellent, good and fair in $78.3 \%$ (90 knees) and poor results in 38 knees (29.7\%). Dhillon et al reported 50\% (50 knees) had poor results and another $50 \%$ good and fair results ${ }^{1}$. They noted that poor results occurred mostly in patients with rheumatoid arthritis and that the knee score improved but the functional score remained poor due to multiple joint diseases. For patients with osteoarthritis, knee scores improved, but the functional score remained poor for cultural reasons. Female patients who have become accustomed to a sedentary way of life confined to their homes, may continue to refuse to walk long distances or attempt to climb stairs. Furthermore a large number do not have stairs in their houses, and some prefer to use a walking stick as it gives them more confidence. These factors reduce their functional score $^{1}$. More than half $(53.1 \%)$ of the rheumatoid arthritis patients, had a poor result and only $21.9 \%$ of osteoarthritis knee had a poor result.

Callahan et al reported that approximately $18 \%$ of patients experienced a postoperative complication following TKA. These included: superficial infections, 3.9\% (range, $0 \%$ to $14.8 \%$ ); deep infections, $1.7 \%$ (range, $0 \%$ to $11.4 \%$ ); pulmonary embolism, $2 \%$ (range, $0 \%$ to $9.7 \%$ ); DVT, $6.5 \%$ (range, $0 \%$ to $56.6 \%$ ); peripheral nerve injury, $2.1 \%$ (range, $0 \%$ to $18.8 \%)^{19}$. Scott et al reported $13.1 \%$ complication post TKA including deep infections, delayed healing ( 3 knees), death, urinary tract infection (2 each), hepatitis (1 knee) and patella fracture (6 knees).[7] In the present study, the incidence of deep infection was 3.9\%, superficial infection $3.9 \%, 0.8 \%$ instability, and $0.8 \%$ thinning of polyethelene. There were no instances of DVT, periprosthetic fracture, pulmonary embolism, neurovascular or peroneal nerve injury. Diduch et al reported: late infection, 2\% (2 of 103 knees); polyethylene thinning, 1\%; dislocation of tibial component, 3\%; instability requiring change of the tibial insert, $1 \%{ }^{14}$. Stern and Insall reported $14(7.7 \%)$ of 180 knees needed revision for various reasons ${ }^{8}$. In the present study, the rate of subluxation due to instability and inadequate size of tibial insert was $0.8 \%$ (1 knee), while Diduch et al also reported 1 incident (1\%) of spacer exchange due to instability.[14] Diduch et al also reported 1 patient $(1 \%)$ had a revision surgery due to polyethylene wear $^{14}$. Schai et al reported worn polyethylene tibial inserts were exchanged in 9 knees $(3.8 \%), 6$ years after the primary operation ${ }^{10}$.

The survival rate at 12 years following TKA in this study was $89.5 \%$. Survivorship analysis is especially useful when not all patients have been at risk for the same period of time. The results of survivorship analysis can depend on the way that the outcome is measured, particularly the end point. A wide variety of end-points have been used. Knutson et al defined end-points as revision with removal of implant and addition or exchange of prosthetic components ${ }^{3}$. Diduch $e t$ al defined the end point as revision of tibial or femoral components (94\% survival rate at 18 years), revision of patella (90\% survival rate at 18 years), and revision of the tibial insert (87\% survival rate at 18 years $)^{14}$. Duffy et al reported a $99 \%$ survival rate at 10 years ${ }^{6}$. Ranawat et al reported asurvival rate of $94.1 \%$ at 15 years ${ }^{12}$. Schai et al (10) obtained $97 \%$ survivorship analysis of the PFCcruciate-retaining arthroplasty system. Scuderi et al reported $97.34 \%$ success rate at 10 years, and also noted that overall survival rate was not influenced by sex or age, diagnosis or percentage of ideal body weight ${ }^{14}$. Rand and Ilstrup reported cumulative survival rates of 9200 total knee arthroplasties performed from 1971 through 1987 at the Mayo Clinic ${ }^{12}$. They presented data showing that in a younger age group (less than 60 years old), the survival rate was $96 \%$ at 2 years, $90 \%$ at 5 years, $76 \%$ at 10 years. For older groups, the rates of survival were $97 \%$ at 2 years, $92 \%$ at 5 years, and $82 \%$ at 10 years. Font-Rodriquez, et al reported In terms of survivorship of cemented TKAs in 2629 knees, FontRodriques reported a 21-year success rate of $90.77 \%$ with an annual failure rate of $0.46 \%$ (215 patients).

\section{CONCLUSIONS}

This study showed good results for total knee replacement surgery in our region during the 1980s to 1990s, and further that the total knee replacement is a reproducible surgery for which a trained surgeon will be able to produce excellent and good results as seen in other studies. 


\section{REFERENCES}

1. Dhillon K, Jamal, Bhupinderjeet S. Early results of Total Knee Replacement. A clinical and radiological evaluation. Med $J$ Malaysia 1993; 48: 427-435.

2. Insall JN, Dorr LD, Scott RD, Scott WN. Rationale of The Knee Society Clinical Rating System. Clin Orthop Relat Res 1989; 248: $13-4$.

3. Knutson K, Lindstrand A, Lidgren L. Survival of knee arthroplasties. A nation-wide multicentre investigation of 800 cases. $J$ Bone Joint Surg 1986; 68B: 795-803.

4. Kaplan EL, Meier P. Nonparametric estimation from incomplete observations. J Am Stat Assoc 1957; 53: 457.

5. Insall JN, Ranawat CS, Aglietti P, Shine J. A Comparison of four models of Total Knee Replacement Prosthesis. J Bone Joint Surg 1976; 58A: 754-65.

6. Rand JA, Ilstrup DM. Survivorship analysis of Total Knee Arthroplasty. Cumulative rates of survival of 9200 total knee arthroplasties. J Bone Joint Surg 1991; 73A: 397-409.

7. Scott WN, Rubinstein M, Scuderi G : Results after knee replacement with a posterior cruciate substituting prosthesis. J Bone Joint Surg 1988; 70A: 1163-73.

8. Stern SH, Insall JN. Posterior Stabilized Prosthesis Results After follow up of nine to twelve years. J Bone and Joint Surg 1992; 74A: 980-6.

9. Nelissen RGHH, Brand R, Rozing PM. Survivorship analysis in Total Condylar Knee Arthroplasty. A Statistical Review. J Bone Joint Surg 1992; 74A: 383-9.

10. Schai PA, Thornhill TS, Scott RD. Total Knee Arthroplasty with the PFC System. Result at a minimum of ten years and survivorship analysis. J Bone Joint Surg 1998; 80B: 850-8.

11. Schurman DJ, Parker JN, Ornstein D. Total Condylar Knee Replacement : A Studyof factors Influencing range of motion as late as two years after arthroplasty. J Bone Joint Surg 1985; 67A: 1006-14.

12. Ranawat CS, Flynn WF, Saddler S, Hansraj KK, Maynard MJ. Long term results of the Total Condylar Knee Arthroplasty : A 15-year survivorship study. Clin Orthop Relat Res 1993; 286: 94-102.

13. Duffy GP, Trousdale RT, Stuart MJ. Total Knee Arthroplasty in patients 55 years old or younger. 10- to 17-year results. Clin Orthop Relat Res 1998; 356: 22-7.

14. Diduch DR, Insall JN, Scott N, Scuderi GR, Font-Rodriquez D. The total knee replacement in young, active patients. Long term follow-up and functional outcome. J Bone Joint Surg 1997; 79A: 575-82.

15. Ewald FC, Jacobs MA, Miegel RE, Walker PS, Poss R, Sledge CB. Kinematic Total Knee Replacement. J Bone and Joint Surg 1984; 66A: 1032-40.

16. Ranawat CS, Rodriquez JA. Malalignment and Malrotation in Total Knee Arthroplasty in Current Concepts in Primary and Revision Total Knee Arthroplasty edited by JN Insall, WN Scott and GR Scuderi Lippincott-Raven Chapter 13. 1996; $115-121$.

17. Ewald FC. The knee society total knee arthroplasty roentgenographic evaluation and scoring system. Clin Orthop Relat Res 1989; 248: 9-15.

18. Brander VA, Malhotra S, Jet J, Heimann AW, Stulberg SD. Outcome of hip and knee arthroplasty in persons aged 80 years and older. Clin Orthop Relat Res 1997; 345: 67-78.

19. Callahan CM, Drake BG, Heck DA, Ditlus RS. Patient outcomes following tricompartmental Total Knee Replacement. A metaanalysis. J Am Med Assoc 1994; 217(17): 1349-57.

20. Scuderi GR, Insall JN, Windsor RE, Moran MC. Survivorship of cemented knee replacements. J Bone and Joint Surg 1989; 71B: 798-803.

21. Font-Rodriquez DE, Scuderi GR, Insall JN. Survivorship of cemented Total Knee Arthroplasty. Clin Orthop Relat Res 1997; 345 : $79-86$. 